\title{
Unknown Combinatorial Auction Mechanisms for Heterogeneous Spectrum Redistribution*
}

\author{
Zhenzhe Zheng ${ }^{\dagger}$, Fan $\mathrm{Wu}^{\dagger}$, Shaojie Tang ${ }^{\S}$, and Guihai Chen ${ }^{\dagger}$ \\ ${ }^{\dagger}$ Shanghai Key Laboratory of Scalable Computing and Systems, Shanghai Jiao Tong University, China \\ ${ }^{\S}$ Department of Information Systems, University of Texas at Dallas, USA \\ \{zhengzhenzhe,wu-fan\}@sjtu.edu.cn, tangshaojie@gmail.com, gchen@cs.sjtu.edu.cn
}

\begin{abstract}
With the growing deployment of wireless communication technologies, radio spectrum is becoming a scarce resource. Auctions are believed to be among the most effective tools to solve or relieve the problem of radio spectrum shortage. However, designing a practical spectrum auction mechanism has to consider five major challenges: strategic behaviors of unknown users, channel heterogeneity, preference diversity, channel spatial reusability, and social welfare maximization. Unfortunately, none of existing work fully considered these five challenges. In this paper, we model the problem of heterogeneous spectrum allocation as a combinatorial auction, and propose AEGIS, which is the first framework of unknown combinatorial Auction mEchanisms for heteroGeneous spectrum redIStribution. AEGIS contains two mechanisms, namely AEGIS-SG and AEGIS-MP. AEGIS-SG is a direct revelation combinatorial spectrum auction mechanism for unknown single-minded users, achieving strategy-proofness and approximately efficient social welfare. We further design an iterative ascending combinatorial auction, namely AEGIS-MP, to adapt to the scenario with unknown multi-minded users. AEGIS-MP is implemented in a set of undominated strategies and has a good approximation ratio. We evaluate AEGIS on two practical datasets: Google Spectrum Database and GoogleWiFi. Evaluation results show that AEGIS achieve much better performance than the state-of-the-art mechanisms.
\end{abstract}

\footnotetext{
${ }^{\dagger} \mathrm{F}$. Wu is the corresponding author.

${ }^{*}$ This work was supported in part by the State Key Development Program for Basic Research of China (973 project 2014CB340303 and 2012CB316201), in part by China NSF grant 61272443 and 61133006 , in part by Shanghai Science and Technology fund 12PJ1404900 and 12ZR1414900, and in part by Program for Changjiang Scholars and Innovative Research Team in University (IRT1158, PCSIRT) China. The opinions, findings, conclusions, and recommendations expressed in this paper are those of the authors and do not necessarily reflect the views of the funding agencies or the government.
}

Permission to make digital or hard copies of all or part of this work for personal or classroom use is granted without fee provided that copies are not made or distributed for profit or commercial advantage and that copies bear this notice and the full citation on the first page. Copyrights for components of this work owned by others than ACM must be honored. Abstracting with credit is permitted. To copy otherwise, or republish, to post on servers or to redistribute to lists, requires prior specific permission and/or a fee. Request permissions from permissions@ acm.org.

MobiHoc'14, August 11-14, 2014, Philadelphia, PA, USA.

Copyright 2014 ACM 978-1-4503-2620-9/14/08 ...\$15.00.

http://dx.doi.org/10.1145/2632951.2632964

\section{Categories and Subject Descriptors}

C.2.1 [Computer-Communication Networks]: Network Architecture and Design - Wireless Communication

\section{Keywords \\ Channel Allocation; Combinatorial Auction}

\section{INTRODUCTION}

The fast development of wireless networks and mobile communications is exhausting the limited radio spectrum resource. However, currently, almost all spectrum is statically allocated to large service providers on a long term basis for large geographical regions, which is reflected in the radio regulations published by the International Telecommunication Union (ITU) [11]. Such static spectrum management leads to low utilization in spatial and temporal dimensions. On one hand, many spectrum owners (i.e., primary users) are willing to lease out their idle spectrum and obtain proper profit. On the other hand, new wireless applications (i.e., secondary users), starving for spectrum, would like to pay for using the spectrum. Therefore, an open and market-based framework is highly needed to redistribute the idle spectrum, and thus improve the utilization of spectrum. Spectrum Bridge [25] is an emerging platform that provides services for buying, selling, and leasing idle spectrum.

Due to the fairness and allocation efficiency, auctions are attractive market-based mechanisms to distribute resources. Examples include FCC spectrum license auctions in the Unite States [6], and auctions for UMTS [16] and LTE [18] in Europe. While these auctions target only at large wireless service providers, our focus is secondary spectrum markets of small wireless applications, such as community wireless networks and home wireless networks.

Designing a feasible and practical spectrum auction has its own challenges. The first major challenge comes from the strategic behaviors of rational and selfish wireless users. In practical spectrum auctions, selfish users can not only misreport their valuations, but also their channel demands, to increase their utilities. We call them unknown users when both the valuations and channel demands are private information. The model of unknown users does not fall into the family of conventional mechanism design with one-parameter domains [1], and has not been considered in any of the existing work for spectrum auctions.

Another design challenge is to consider both the channel heterogeneity and preference diversity. The channel heterogeneity comes from both spatial heterogeneity and frequency heterogeneity. On one hand, the availability and quality of spectrum vary at different locations. On the other hand, spectrum resided in different frequency bands may have different propagation and penetration characteristics. Due to 


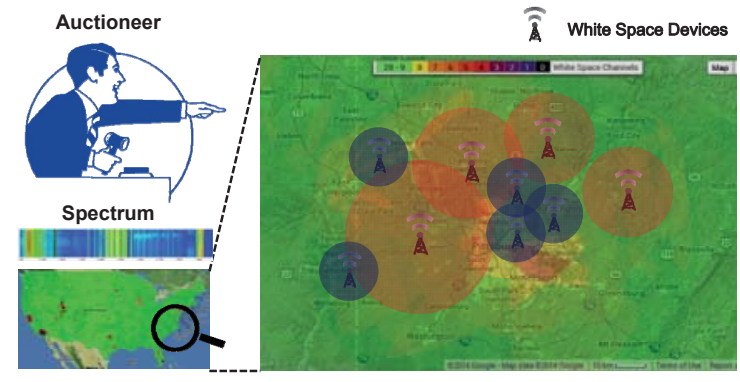

Figure 1: Heterogeneous TV White Space spectrum redistribution based on combinatorial auctions.

the heterogeneity of channels, users may have diverse preferences on different combinations of heterogeneous channels. For instance, a secondary user may be likely to have a valuation for some paired channels to provide LTE-based services, and have another different valuation for unpaired channels to support WiMax services. Therefore, it is necessary to allow users to express different valuations on multiple channel bundles. Considering the channel heterogeneity and preference diversity, it is natural to model the market of heterogeneous spectrum redistribution as a combinatorial auction. However, spectrum is different from traditional goods due to its spatial reusability, by which well-separated users can be allocated on the same spectrum band simultaneously. Thus, traditional combinatorial auction mechanisms cannot be directly applied to spectrum auctions. Figure 1 shows a combinatorial auction mechanism for TV white space spectrum redistribution. The TV white spaces has both spatially and frequency heterogeneity. White space devices at different locations can access to different available white spaces, and have distinct permissive maximum power, adjacent channel interference and noise floor. The frequency of TV broadband ranges from $54 \mathrm{MHz}$ to $806 \mathrm{Mhz}$, leading to various frequency characteristics of different white spaces.

The last but not least design challenge is the basic and common objective of auctions: maximizing social welfare, which is defined as the sum of winners' valuations on allocated goods (Please refer to Section 3.2 for the definition). However, finding the optimal social welfare in combinatorial spectrum auctions is normally computationally intractable.

In this paper, we conduct an in-depth study on the problem of dynamic spectrum redistribution, jointly considering the above challenges. We propose a family of unknown combinatorial Auction mEchanisms for heteroGeneous spectrum redIStribution (AEGIS). AEGIS contains two mechanisms, namely AEGIS-SG and AEGIS-MP. Specifically, AEGIS-SG is a direct revelation combinatorial auction for unknown single minded users, achieving both strategy-proofness (Please refer to Section 3.3 for the definition) and a good approximation ratio. AEGIS-MP is a novel iterative ascending combinatorial auction for unknown multiple minded users. AEGIS-MP achieves approximately efficient social welfare, and is implemented in undominated strategies, which is an important solution concept from game theory (Please refer to Section 3.3 for the definition). To the best of our knowledge, AEGIS is the first combinatorial auction framework considering spectrum redistribution among unknown users.

We summarize our contributions in this paper as follows.

- First, we propose a general combinatorial auction model for the problem of heterogeneous spectrum redistribution, and use the concept of virtual channel to capture the conflict of channel usage among wireless users. This general model is powerful enough to express channel heterogeneity and spatial reusability, as well as preference diversity.

- Second, we begin with considering a simple but classical setting with unknown single minded users, and propose AEGIS-SG, which is a strategy-proof and approximately efficient combinatorial auction mechanism for heterogeneous channel redistribution.

- Third, we further extend this work by considering a more general case, in which users are unknown multiple minded. We propose a novel iterative ascending combinatorial auction mechanism, namely AEGIS-MP, which is an algorithmic implementation in undominated strategies, and achieves approximately efficient social welfare.

- Last but not least, we evaluate the performance of AEGIS based on two practical datasets, Google Spectrum Database and GoogleWiFi, and compare AEGIS with the state-of-theart mechanisms. Our evaluation results show that AEGIS achieves superior performance in terms of social welfare, revenue, user satisfaction ratio, and channel utilization.

The rest of this paper is organized as follows. In Section 2, we review related work. In Section 3, we present the model of combinatorial auction for heterogeneous spectrum redistribution. In Section 4, we propose AEGIS-SG for the case with unknown single minded users. We further consider the case with unknown multiple minded users, and propose AEGIS-MP in Section 5. The evaluation results are presented in Section 6. We conclude the paper in Section 7.

\section{RELATED WORK}

In recent years, designing auction mechanisms for spectrum redistribution attracts increasing interests $[5,7,29,31$, 33]. Unfortunately, none of these mechanisms fully consider the above design challenges. Some of the spectrum auction mechanisms (e.g., VERITAS [31] and TRUST [33]) consider channel spatial reusability, but fail in heterogeneous channel scenarios. Recent work CRWDP [5], TAHES [7] and SMASHER [29] consider channel heterogeneity, but CRWDP ignores channel spatial reusability, while TAHES and SMASHER have simple valuation formats. Furthermore, these mechanisms only prevent users from misreporting their valuations to manipulate the auction, and always assume that the channel demands are publicly known to the auctioneer. However, in practice, users can further improve their utilities by cheating on their channel demands. In this work, we design heterogeneous spectrum auction mechanisms, considering both channel spatial reusability and diverse valuation formats. To some extent, our mechanisms are resistant to both valuations and channel demands cheating behaviors.

There are some other related work on spectrum auctions mechanism design, e.g., online spectrum auction [28], revenue generation [15] and spectrum auction with multiple auctioneers [8] Besides auction theory, some other powerful tools, e.g., contract theory [24], queueing theory [13], and randomized algorithm [10], have been applied to different scopes in spectrum markets design.

Another category of related work is combinatorial auction mechanism design. Dobzinski [4] and Papadimitriou et al. [23] proved that optimal social welfare and strategyproofness cannot be achieved simultaneously in general combinatorial auctions. Considering the intractability of combinatorial auctions, a number of strategy-proof auction mechanisms with well bounded approximation ratios are proposed $[1,3,20]$. There are still no positive results (computationally efficient, deterministic and strategy-proof mechanisms with good social welfare approximation) to combinatorial auctions with unknown multi-minded buyers. Our design is based on the iterative wrapper technique for unknown combinatorial auction mechanism [2]. However, none 
of the above combinatorial auctions considered the spectrum spatial reusability.

\section{PRELIMINARIES AND PROBLEM FOR- MULATION}

In this section, we first describe network and auction model for the problem of heterogeneous channel redistribution, and then review related solution concepts used in this paper from game theory. At last, we formulate the channel allocation problem as a classic weighted set packing problem.

\subsection{Network Model}

We consider a static secondary spectrum market with a primary spectrum holder, called "seller", and some secondary users (e.g., WiFi APs), called "buyers". The primary spectrum holder wants to sell her temporary unused spectrum, and the secondary users would like to lease spectrum to provide wireless services for their customers at certain Quality of Service (QoS). We consider that the trading channels are heterogeneous, and thus buyers have diverse preferences over the different combinations of channels according to their QoS, hardware abilities and the interference conditions of accessible channels. Different from traditional goods, wireless channels can be spatially reused, meaning that conflict-free buyers can be allocated the same channel simultaneously.

We denote the set of $m$ orthogonal and heterogeneous channels for leasing by $\mathbb{C} \triangleq\left\{c_{1}, c_{2}, \ldots, c_{m}\right\}$, and the set of buyers by $\mathbb{N} \triangleq\{1,2, \ldots, n\}$.

Conflict Graph: In spectrum auctions, conflict graphs are usually used to represent the interference among buyers, and can be built by the auctioneer through some measurement methods, e.g., measurement calibrated method [32]. Due to the heterogeneity of channels, each channel may have a distinct conflict graph. Let $\mathcal{G}_{k} \triangleq\left(\mathcal{N}_{k}, \mathcal{E}_{k}\right)$ denote the conflict graph on channel $c_{k}$, where $\mathcal{N}_{k} \subseteq \mathbb{N}$ is the set of buyers who can access channel $c_{k}$, and each edge $(i, j) \in \mathcal{E}_{k}$ represents the interference between buyers $i$ and $j$ on channel $c_{k}$. We also denote the maximum degree on graph $\mathcal{G}_{k}$ by $\Delta_{k}$, and the maximum among all $\Delta_{k}$ 's by $\Delta_{\max }$, i.e., $\Delta_{\max } \triangleq \max _{c_{k} \in \mathbb{C}}\left\{\Delta_{k}\right\}$.

\subsection{Auction Model}

We model the process of heterogeneous channels redistribution as combinatorial auctions. We discuss two popular kinds of combinatorial auctions: direct revelation combinatorial auction for the case with unknown single-minded buyers, and iterative ascending combinatorial auction for the case with unknown multi-minded buyers. Specifically, in the direct revelation combinatorial auction, buyers simultaneously declare their bids and channel demands to a trustworthy auctioneer, and then the auctioneer makes the decision on channel allocation and the charge to each winner. In the iterative ascending combinatorial auction, buyers compete by gradually raising their bids, and the auctioneer maintains a provisional allocation in each iteration. The auction stops when all the remaining active buyers are declared as winners, and winners pay their lastly reported bids. We list some useful notations in our model as follows.

Interested Channel Bundle: Each buyer $i \in \mathbb{N}$ has various private preferences on $l_{i}$ channel bundles $\hat{\mathbf{S}}_{i} \triangleq\left\{\hat{S}_{i}^{1}, \hat{S}_{i}^{2}, \ldots\right.$, $\left.\hat{S}_{i}^{l_{i}}\right\}$, in which $\hat{S}_{i}^{j} \subseteq \mathbb{C}$ and $l_{i}$ can be arbitrarily large, even exponential. We call a buyer $i$, who is interested in $l_{i}$ channel bundles as $l_{i}$-minded buyer. We discuss single-minded case $\left(l_{i}=1\right.$ for all $\left.i \in \mathbb{N}\right)$ in Section 4 and multi-minded case $\left(l_{i}>\right.$ 1 for some $i \in \mathbb{N}$ ) in Section 5. We denote the interested bundles of all buyers by $\mathbb{S} \triangleq\left\{\hat{\mathbf{S}}_{1}, \hat{\mathbf{S}}_{2}, \cdots, \hat{\mathbf{S}}_{n}\right\}$.
Valuation: Each buyer $i \in \mathbb{N}$ has a private valuation $v_{i}^{j}$ over each of her interested channel bundle $\hat{S}_{i}^{j} \in \hat{\mathbf{S}}_{i}$. For the other channel bundles not in $\hat{\mathbf{S}}_{i}$, we adopt the XOR operation in combinatorial auctions [21], and formally describe the valuation function of buyer $i$ as

$$
V_{i}(S) \triangleq \begin{cases}\max _{\hat{S}_{i}^{j} \in \hat{\mathbf{S}}_{i}, \hat{S}_{i}^{j} \subseteq S}\left\{v_{i}^{j}\right\}, & \exists \hat{S}_{i}^{j} \in \hat{\mathbf{S}}_{i}, \hat{S}_{i}^{j} \subseteq S, \\ 0, & \text { otherwise. }\end{cases}
$$

We assume that $V_{i}(\cdot)$ is normalized (i.e., $V_{i}(\varnothing)=0$ ) and monotone (i.e., $V_{i}(S) \leq V_{i}(T)$ for each $S \subseteq T \subseteq \mathbb{C}$ ). Since the valuation function is derived from the expected quality of wireless service applications, we can assume that the range of the valuation function $V_{i}(\cdot)$ of buyer $i$ is $\left[\underline{v}_{i}, \bar{v}_{i}\right]$ and $v_{i} \geq \epsilon$, where $\epsilon$ is the minimum monetary unit in auction systems. We denote the maximum value of all $\bar{v}_{i}$ by $\bar{v}_{\text {max }} \triangleq \max _{i \in \mathbb{N}} \bar{v}_{i}$. We call the valuation function of buyer $i$ is $\delta_{i}$-close when $\frac{\bar{v}_{i}}{\underline{v}_{i}} \leq \delta_{i}$. This parameter characterizes the diversity of valuation function from one buyer. Let $\delta_{\text {max }} \triangleq \max _{i \in \mathbb{N}} \delta_{i}$. We denote the valuation functions of all buyers by $\overrightarrow{\mathcal{V}} \triangleq\left(V_{1}(\cdot), V_{2}(\cdot), \cdots, V_{n}(\cdot)\right)$.

Bid and Declared Channel Bundle: In the direct revelation combinatorial auction for unknown single-minded case, each buyer $i \in \mathbb{N}$ declares a bid $b_{i}$ and one channel bundle $\mathbf{S}_{i}$ to the auctioneer, meaning that she is willing to pay at most $b_{i}$, if she is allocated a channel bundle containing $\mathbf{S}_{i}$. The bid vector of all buyers is represented as $\overrightarrow{\mathcal{B}} \triangleq\left(b_{1}, b_{2}, \cdots, b_{n}\right)$, and the declared channel bundles of all buyers are denoted by $\overrightarrow{\mathcal{S}} \triangleq\left(\mathbf{S}_{1}, \mathbf{S}_{2}, \cdots, \mathbf{S}_{n}\right)$. In the iterative ascending combinatorial auction for unknown multi-minded case, active buyer $i$ submits a temporary bid $b_{i}^{j}$ and a channel bundle $\mathbf{S}_{i}^{j}$ in the $j$ th iteration. The bids of non-active buyers are set to zeros, and their current bundles are the declared bundles when they drop out of the auction. We denote all bids by $\overrightarrow{\mathcal{B}}^{j} \triangleq\left(b_{1}^{j}, b_{2}^{j}, \cdots, b_{n}^{j}\right)$ and the declared bundles of buyers by $\overrightarrow{\mathcal{S}}^{j} \triangleq\left(\mathbf{S}_{1}^{j}, \mathbf{S}_{2}^{j}, \cdots, \mathbf{S}_{n}^{j}\right)$ in the $j$ th iteration.

Clearing Price and Utility: The auctioneer charges each winner $i$ a clearing price $p_{i}$, and let the losers free of any charge. We use vector $\overrightarrow{\mathcal{P}} \triangleq\left(p_{1}, p_{2}, \cdots, p_{n}\right)$ to represent the clearing prices of all buyers. Each buyer $i \in \mathbb{N}$ has a quasilinear utility defined as

$$
u_{i} \triangleq V_{i}\left(\mathbf{S}_{i}^{*}\right)-p_{i},
$$

where $\mathbf{S}_{i}^{*}$ is the channel bundle allocated to buyer $i$.

In our model, we consider buyers are unknown, i.e., both the valuation functions and interested channel bundles are private information, and unknown to the auctioneer. In such environment, the selfish and rational buyers have more flexibilities to manipulate the results of the auction, and are eager to maximize their own utilities. In contrast to buyers, the overall objective of the auction mechanism is to maximize social welfare, which is defined as follows.

Definition 1 (Social Welfare). The social welfare in a spectrum auction is the sum of winning buyers' valuations on their allocated bundles of channels, i.e.,

$$
S W \triangleq \sum_{i \in \mathbb{W}^{*}} V_{i}\left(\boldsymbol{S}_{i}^{*}\right)
$$

where $\mathbb{W}^{*}$ is the set of winners, and $\boldsymbol{S}_{i}^{*}$ is the corresponding allocated channel bundle for winner $i$.

In this paper, we assume that buyers do not collude with each other, and leave relaxation of this assumption to our future work. 


\subsection{Economic Properties}

We briefly review the solution concepts used in this paper from game theory.

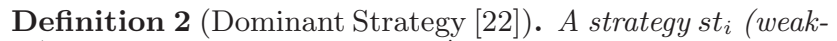
ly) dominates another strategy st ${ }_{i}^{\prime}$ of player $i$, if for any other players' strategy profile $s t_{-i}: u_{i}\left(s t_{i}, s t_{-i}\right) \geq u_{i}\left(s t_{i}^{\prime}, s t_{-i}\right)$, and this inequality is strict for at least one instance of $s t_{-i}$.

A strategy $s_{i}$ is a dominant strategy for player $i$ if it (weakly) dominates any other strategies of player $i$.

A strategy $s t_{i}$ is an undominated strategy for player $i$ if it is not dominated by any other strategies of player $i$.

In direct revelation mechanisms, incentive-compatibility means that truthfully revealing private information (both the valuations and channel demands in this paper) is a dominant strategy for each player. An accompanying concept is individual-rationality, which means that players truthfully participating in the game gain non-negative utilities. The formal definition of strategy-proof mechanism is as follows.

Definition 3 (Strategy-Proof Mechanism [19]). A direct revelation mechanism is strategy-proof when it satisfies both incentive-compatibility and individual-rationality.

Strategy-proofness is a strong solution concept in mechanism design. However, the requirement of having dominant strategies limits the existence of feasible allocation algorithms in combinatorial auctions for unknown multi-minded buyers [3,17]. Therefore, we turn our attention to another well-known game theoretic concept: implementation in undominated strategies.

Definition 4 (Implementation in Undominated Strategies $[2,12])$. A mechanism $\mathcal{M}$ is an implementation of c-approximation in undominated strategies if there exists a non-empty set of undominated strategies D with the following properties.

- $\mathcal{M}$ achieves a c-approximation in polynomial time for any combination of undominated strategies from $D .{ }^{1}$

- $\mathcal{M}$ is individually rational for players taking undominated strategies from $D$.

- $\mathcal{M}$ has fast undominance recognition property, meaning that a player can efficiently determine if a strategy belongs to $D$, and if not, compute an undominated strategy in $D$ to dominate it.

The underlying goal of spectrum auctions is to achieve approximately optimal social welfare in the present of strategic behaviors of buyers. In unknown multi-minded case, we achieve this goal by relaxing the strict strategy-proofness constraint, and allowing the mechanism to leave several strategies in $D$ for the buyers to choose from. We compensate this uncertainly on the game-theoretic side by strengthening the algorithmic analysis, showing that the mechanism can achieve a good approximation for any combination of undominated strategies from $D$.

\subsection{Problem Formulation}

We borrow the novel concept of virtual channel [29] to represent the conflict of channel usage among buyers. By using virtual channels, we transform the channel allocation problem to a classical weighted set packing problem, and formulate it as a binary program. Specifically, a virtual channel $h_{i, j}^{k}$ indicates that buyers $i$ and $j$ cause interference between each other on channel $c_{k}$, when channel $c_{k}$ is allocated to $i$

\footnotetext{
${ }^{1}$ In this paper, a mechanism $\mathcal{M}$ achieves $c$-approximation means that the approximation ratio of $\mathcal{M}$ is $\frac{1}{c}$. The approximation ratio is defined as the ratio between the social welfare achieved by $\mathcal{M}$ and the optimal social welfare.
}

and $j$ simultaneously, i.e., virtual channel $h_{i, j}^{k}$ is corresponding to the edge $(i, j) \in \mathcal{E}_{k}$ on conflict graph $\mathcal{G}_{k}$. We now show the process of constructing virtual channels. We first create virtual channel $h_{i, j}^{k}$ for each edge $(i, j) \in \mathcal{E}_{k}$ on conflict graph $\mathcal{G}_{k}$, and then append $h_{i, j}^{k}$ to the channel bundles containing channel $c_{k}$ from the buyers $i$ and $j$. We finally remove the original channels from all channel bundles. Hence, the updated channel bundles only contain virtual channels. From now on, the set $\mathbb{S}$ and vector $\overrightarrow{\mathcal{S}}\left(\overrightarrow{\mathcal{S}}^{j}\right)$ represent the updated interested channel bundles and updated declared channel bundles, respectively. We note that the valuations on updated channel bundles retain the same. Let $H_{i}$ be the set of virtual channels for the buyer $i$. All virtual channels are denoted by $\mathbb{H} \triangleq\left\{H_{1}, H_{2}, \cdots, H_{n}\right\}$. According to the rule of virtual channel construction, the maximum size of updated interested channel bundle is bounded by $\kappa=m \times \Delta_{\max }$.

When virtual channel $h_{i, j}^{k}$ is added into the channel bundles containing channel $c_{k}$ from the buyers $i$ and $j$, at most one of the channel bundles from the buyers $i$ and $j$ can be allocated, ensuring the exclusive allocation of channel $c_{k}$ for the buyers $i$ and $j$. If the buyer $i$ obtains all virtual channels $h_{i, j}^{k},(i, j) \in \mathcal{E}_{k}$ on conflict graph $\mathcal{G}_{k}$, then she is granted channel $c_{k}$. We note that the buyer $i$ may not conflict with any other buyers on some channels. For these channels with no interference, we directly allocate them to the buyer $i$. Consequently, the exclusive allocation of virtual channels implies the feasible channel allocation under conflict graph constraint.

We now transform the channel allocation problem to the weighted $\kappa$-set packing problem. The weighted $\kappa$-set packing problem can be described as: given a family of weighted sets, each containing at most $\kappa$ elements drawn from a finite universe, find a maximum weight sub-collection of disjoint sets. In the channel allocation problem, the set of virtual channels $\mathbb{H}$ corresponds to the universe, collection of updated declared channel bundles $\overrightarrow{\mathcal{S}}\left(\overrightarrow{\mathcal{S}}^{j}\right)$ corresponds to the family of weighted sets, and $\kappa=m \times \Delta_{\max }$. In the direct revelation combinatorial auction for the single-minded case, the problem of channel allocation can be formulated as an integer programming.

Problem: Heterogeneous Channel Allocation
Objective: Maximize $\sum_{i \in \mathbb{N}}\left(x\left(i, \mathbf{S}_{i}\right) \times b_{i}\right)$
Subject to:
\[ \sum_{i \in \mathbb{N}} \sum_{\mathbf{S}_{i} \ni h_{k}} x\left(i, \mathbf{S}_{i}\right) \leq 1, \quad \forall h_{k} \in \mathbb{H} \]
$x\left(i, \mathbf{S}_{i}\right) \in\{0,1\}, \quad \forall i \in \mathbb{N}$

Here, the variable $x\left(i, \mathbf{S}_{i}\right)=1$ indicates that channel bundle $\mathbf{S}_{i}$ is allocated to buyer $i$; otherwise $x\left(i, \mathbf{S}_{i}\right)=0$. The first set of constraints represents the exclusive allocation of virtual channels, and the second set of constraints states the binary value of the auctioneer's decision of allocation. In the $j$ th iteration of ascending combinatorial auction, we can formulate the channel allocation problem as a similar integer programming by replacing $b_{i}$ and $\mathbf{S}_{i}$ with $b_{i}^{j}$ and $\mathbf{S}_{i}^{j}$. In the formulation, we use declared information $(\overrightarrow{\mathcal{B}}, \vec{S}$, $\overrightarrow{\mathcal{B}}^{j}$ and $\vec{S}^{j}$ ) instead of truly private information ( $\mathbb{S}$ and $\overrightarrow{\mathcal{V}}$ ), because the strategy-proof mechanism in Section 4 will guarantee that bidding truthfully is a dominant strategy for each buyer, and the iterative auction mechanism implemented in undominated strategies in Section 5 will ensure that the declared information is close to the truthful information at the end of the auction.

Solving the above integer programming is NP-hard, which makes the general and celebrated VCG mechanism [22] inapplicable. Considering the computational intractability of 


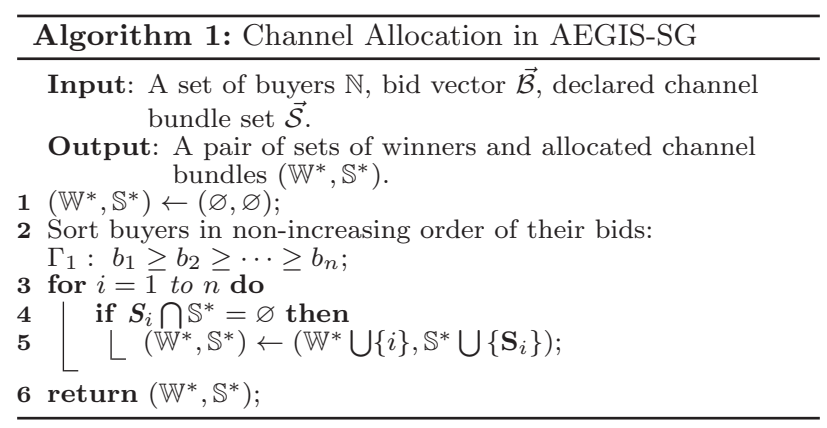

the problem, we present alternative solutions with greedy allocation algorithms to achieve approximately efficient social welfare in following sections.

\section{AEGIS-SG}

In this section, we begin with a simple but classical setting, in which buyers are unknown single-minded. As shown in section 3.4, finding the optimal auction decision is computationally intractable, even in this restricted case. Therefore, we design AEGIS-SG, which is a direct revelation combinatorial auction mechanism for heterogeneous channel redistribution among unknown single-minded buyers, achieving both strategy-proofness and approximate efficiency.

\subsection{Design Details}

We first formally define the concept of unknown singleminded buyers.

Definition 5 (Unknown Single-Minded Buyer). Buyer $i$ is an unknown single-minded buyer iff she is only interested in one channel bundle $\hat{\boldsymbol{S}}_{i} \subseteq \mathbb{H}$, and has a valuation $v_{i}$ for any bundle containing $\hat{\boldsymbol{S}}_{i}$. Both the valuation $v_{i}$ and channel demand $\hat{\boldsymbol{S}}_{i}$ are private information.

AEGIS-SG consists of two major components: greedy channel allocation and clearing price calculation. The greedy channel allocation procedure is depicted in Algorithm 1. The algorithm contains two steps:

- Step 1: We sort buyers according to their bids in nonincreasing order, and denote the sorted list by $\Gamma_{1}$. We break the tie following any bid-independent rule, e.g., lexicographic order of buyers' IDs or channel numbers.

- Step 2: Following the order in $\Gamma_{1}$, we greedily grant channel bundles, which do not overlap with the previous allocated virtual channels.

The clearing price calculation is based on critical bid.

Definition 6 (Critical Bid). The critical bid for buyer $i \in \mathbb{N}$ is the minimum bid that the buyer $i$ should declare to win the auction.

The critical bid of winner $i \in \mathbb{W}^{*}$ can be calculated by the following steps. Consider the winner $i$ in the sorted list $\Gamma_{1}$, we find the first buyer following $i$ that has been denied but would have been granted a channel bundle when the buyer $i$ is removed from $\Gamma_{1}$, and denote this buyer by $\pi(i)$. We note that such a buyer necessarily conflicts with $i$. We can formally represent $\pi(i)$ as

$$
\begin{aligned}
& \pi(i) \triangleq \min \left\{j \mid j>i, \mathbf{S}_{j} \cap \mathbf{S}_{i} \neq \varnothing\right. \text { and } \\
& \left.\quad \forall k<j, k \neq i, k \text { is a winner } \Rightarrow \mathbf{S}_{k} \cap \mathbf{S}_{j}=\varnothing\right\} .
\end{aligned}
$$

The critical bid for the winner $i$ is $b_{\pi(i)}$. We show the method of calculating the clearing price for buyer $i \in \mathbb{N}$ by distinguishing two cases.

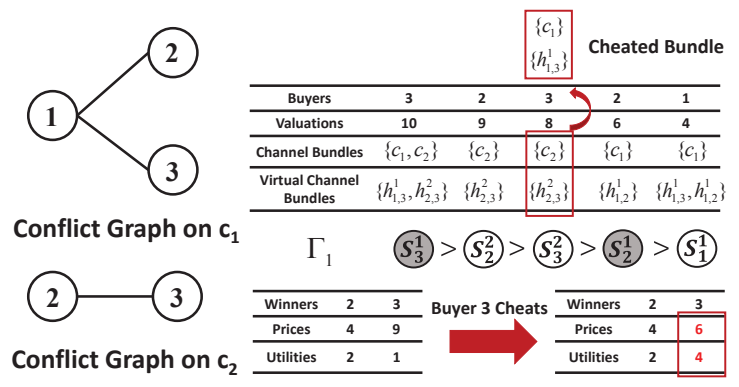

Figure 2: An illustrative example on why extending AEGIS-SG is not strategy-proof in multi-minded scenario. When buyer 3 changes her second interested bundle from $\left\{c_{2}\right\}$ to $\left\{c_{1}\right\}$, she decreases her clearing price from 9 to 6 , and obtains higher utility.

- If buyer $i$ is a loser or $\pi(i)$ does not exist, she pays zero.

- If there exists a $\pi(i)$, and $i$ is granted $\mathbf{S}_{i}$, she pays $b_{\pi(i)}$.

\subsection{Analysis}

In this section, we prove that AEGIS-SG guarantees strategy-proofness in terms of valuations and channel demands, and analyze the approximation ratio of AEGIS-SG. Due to the space limitation, we leave the detailed proofs of these results to our technical report [30].

We first show the monotonicity of channel allocation algorithm, which is essential for a strategy-proof mechanism.

Lemma 1. AEGIS-SG's channel allocation algorithm is monotonic, i.e., buyer $i$, who wins by declaring $\left(\boldsymbol{S}_{i}, b_{i}\right)$, also wins if she declares $\left(\boldsymbol{S}_{i}^{\prime}, b_{i}^{\prime}\right)$, such that, $\boldsymbol{S}_{i}^{\prime} \subseteq \boldsymbol{S}_{i}$ and $b_{i}^{\prime} \geq b_{i}$.

We present the strategy-proofness and approximation ratio of AEGIS-SG.

Theorem 1. AEGIS-SG is a strategy-proof combinatorial spectrum auction for unknown single-minded buyers.

Theorem 2. AEGIS-SG achieves $\mathcal{O}(\kappa)$-approximation.

\section{AEGIS-MP}

In this section, we consider a more general scenario, in which buyers are unknown multi-minded. We first give an illustrative example to show that simply extending AEGISSG can no longer guarantee strategy-proofness. Furthermore, designing a deterministic, approximately efficient and strategy-proof combinatorial auction mechanism for unknown multi-minded buyers is still an open problem in algorithmic mechanism design, and some negative results are demonstrated $[4,23]$. We turn to another well known game theoretic concept, implementation in undominated strategies, and design AEGIS-MP, which is an approximately efficient ascending combinatorial auction for heterogeneous channel redistribution among unknown multi-minded buyers.

We first give the definition of unknown multi-minded buyer.

Definition 7 (Unknown Multi-Minded Buyer). Buyer $i$ is an unknown multi-minded buyer iff she is interested in multiple channel bundles $\hat{\boldsymbol{S}}_{i}=\left\{\hat{S}_{i}^{1}, \hat{S}_{i}^{2}, \cdots, \hat{S}_{i}^{l_{i}}\right\}, l_{i}>1$, and has valuation function $V_{i}(\cdot)$ defined as Equation (1). The channel demands and valuation function are private information.

\subsection{A Counter Example}

In Figure 2, there are three buyers $\{1,2,3\}$, and two trading channels $\left\{c_{1}, c_{2}\right\}$. Since buyers are multi-minded, they 
may have different valuations on different bundles, e.g., buyer 3 has valuation 10 and 8 over channel bundles $\left\{c_{1}, c_{2}\right\}$ and $\left\{c_{2}\right\}$, respectively. Based on the conflict graphs and buyers' interested channel bundles, we can construct virtual channel bundles for each buyer. In this example, we assume that buyers truthfully reveal their valuations, and investigate their manipulated strategies on channel demands.

In AEGIS-SG, we sort buyers' declared channel bundles according to the non-increasing order of their bids, and greedily grant channel bundles, ensuring the exclusive allocation of virtual channels. Buyers 2 and 3 are the winners, and obtain channel bundles $\mathbf{S}_{2}^{1}=\left\{c_{2}\right\}$ and $\mathbf{S}_{3}^{1}=\left\{c_{1}, c_{2}\right\}$, respectively. When bidding truthfully, according to the pricing scheme of AEGIS-SG, buyer 3 should pay 9, which is the bid of buyer 2 on bundle $\mathbf{S}_{2}^{2}$, and her utility is $10-9=1$. However, buyer 3 can cheat by changing her second interested bundle $\mathbf{S}_{3}^{2}$ to $\left\{c_{1}\right\}$, and will still be allocated bundle $\mathbf{S}_{3}^{1}$ but be charged with 6 , increasing her utility to 4 . Hence, by declaring untruthful channel demands, buyers can improve their utilities, which leads to the untruthfulness of AEGISSG in multi-minded scenario.

\subsection{Design Rational}

AEGIS-MP is an ascending Japanese auction [14] on top of a greedy channel allocation algorithm. In traditional ascending Japanese auctions, the auctioneer collects temporary bids from active buyers, and maintains a provisional allocation in each iteration. Provisional losers can choose to increase their bids or permanently drop out of the auction. This process is iterated until all remaining active buyers are winners, and their prices are lastly reported bids.

The most challenging part of designing combinatorial auctions for unknown multi-minded buyers is that both the valuations and channel demands are private and unknown to the auctioneer. We overcome this challenge by extending the ascending Japanese auctions to approach the true valuations and channel demands of buyers. Informally, in AEGIS-MP, we also maintain an "active bundle" for each buyer. This active bundle will keep approaching to one of the interested bundles of the buyer during the auction. Another challenge is the impact of manipulative behaviors of selfish buyers, which should be prevented to form a relatively stable market. Since the buyers are rational, they will not take dominated strategies if some undominated strategies can be quickly recognized. By exploiting this rationality of buyers, we carefully design the structure of auctions, such that, at each decision point, buyers can efficiently recognize the undominated strategies and take one of them, leading AEGIS-MP to be implemented in undominated strategies.

\subsection{Design Details}

We now describe AEGIS-MP in detail. We suppose that GDY_ALG is the approximately efficient greedy-based allocation algorithm, and when given as input vectors of active bundles and temporary bids, it outputs a provisional allocation that is approximate to the optimal solution. In AEGISMP, which is shown in Algorithm 2, the vector of bids $\overrightarrow{\mathcal{B}}^{0}$ and active bundles $\overrightarrow{\mathcal{S}}^{0}$ are initialized to $\vec{\epsilon}$ and $\left(H_{1}, H_{2}, \cdots, H_{n}\right)$, respectively (Line 2). At the beginning of the $j$ th iteration, the auctioneer knows four parts of information: the previous losers set $\mathbb{L}^{j}$, the previous winners set $\mathbb{W}^{j}$, the current active bundle vector $\overrightarrow{\mathcal{S}}^{j}$ and the temporary bid vector of buyers $\overrightarrow{\mathcal{B}^{j}}$. These parameters are handed in as input to GDY_ALG, who, in return, outputs a new set of provisional winners $\mathbb{W}^{j+1}$ and active bundles $\overrightarrow{\mathcal{S}}^{j+1}$ (Line 4 ). The provisional winners retain the same bids, while provisional losers are required to either increase their current bids by multiplying $e$ or permanently drop out of the auction (this is denoted

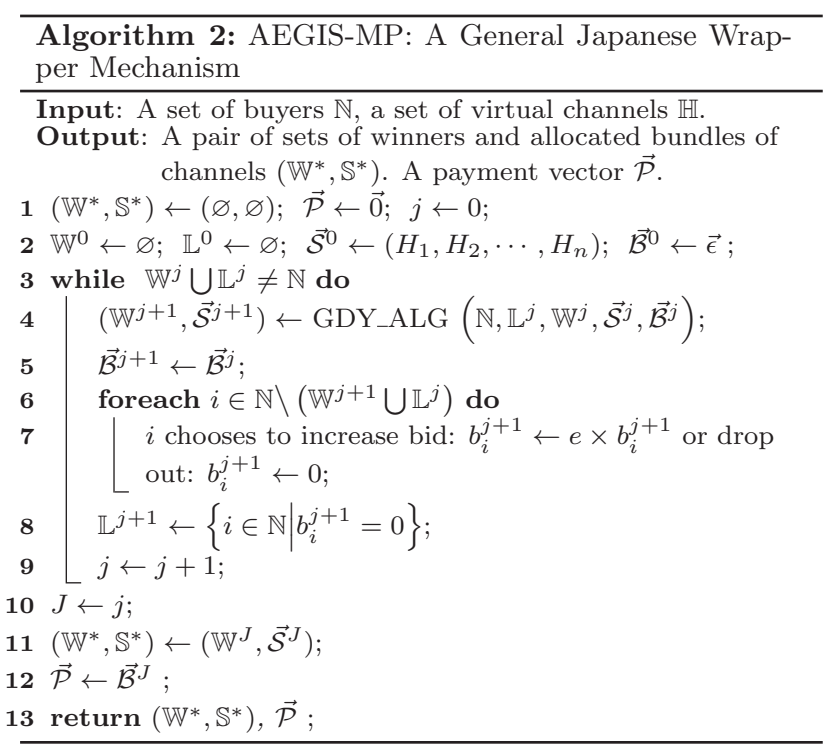

by setting $\left.b_{i}^{j+1}=0\right)($ Lines 5 to 8$)$. Here, parameter $e$ is Euler's Number, and is the best choice over all constants for the optimal approximation ratio. This process is iterated until all remaining active buyers are declared as winners by GDY_ALG. Let the total number of iterations be $J$, and the set of winners is $\mathbb{W}^{J}$. Each winner $i \in \mathbb{W}^{J}$ gets her finally active bundle $\mathbf{S}_{i}^{J} \in \overrightarrow{\mathcal{S}}^{J}$, and pays her lastly reported bid $b_{i}^{J} \in \overrightarrow{\mathcal{B}}^{J}$. The losers will not be allocated bundles, and are free of any charge (Line 11 to 12).

We now depict the design of channel allocation algorithm GDY_ALG in details. Algorithm 3 shows the pseudo-code of GDY_ALG procedure. Let $\mathbb{N}^{j}$ denote the active buyers in the $j$ th iteration. Function $\operatorname{Free}\left(\mathcal{N}, \overrightarrow{\mathcal{S}}^{j}\right)$ denotes the virtual channels not in $\bigcup_{i \in \mathcal{N}} \mathbf{S}_{i}^{j}$. Here, $\mathcal{N} \subseteq \mathbb{N}$ is a subset of buyers, and $\overrightarrow{\mathcal{S}^{j}}$ is a vector of active bundles. GDY_ALG constructs a greedy allocation, Greedy ${ }^{j+1}$, by extending the channel allocation algorithm in AEGIS-SG. Similarly, we sort the active buyers according to their current bids in non-increasing order, and break the tie following any bid-independent rule (Line 2). Following order $\Gamma_{2}$, two steps are performed for the currently considered buyer $i$.

- Shrinking Active Bundle: If the buyer $i$ was previously a provisional loser, then she is given an option to "shrink" her active bundle. If the buyer $i$ chooses to shrink her bundle, the new bundle must satisfy that its valuation is not less than her current bid $b_{i}^{j}$, and it is a subset of the previously reported bundle $\mathbf{S}_{i}^{j}$ and disjoints from the bundles of buyers that are already in Greedy ${ }^{j+1}$ (Line 4 to 5).

- Updating Candidate Winner Sets: Buyer $i$ is added to the allocation Greedy ${ }^{j+1}$ (or $\mathbb{W}^{j}$ ) when her declared bundle $\mathbf{S}_{i}^{j+1}$ does not intersect the bundles of existing buyers in Greedy ${ }^{j+1}$ (or $\mathbb{W}^{j}$ ). This operation ensures that the two allocations Greedy $y^{j+1}$ and $\mathbb{W}^{j}$ are Pareto-efficient ${ }^{2}$ with respect to the new active bundles $\overrightarrow{\mathcal{S}}^{j+1}$ (Line 6 to 7 ).

Once all the active buyers have been considered, GDY_ALG outputs the allocation with the maximum value out of the two allocations Greed $y^{j+1}$ and $\mathbb{W}^{j}$ as the new set of pro-

${ }^{2}$ Pareto-efficiency means that it is impossible to add losers into the winner set without removing at least one winner. 


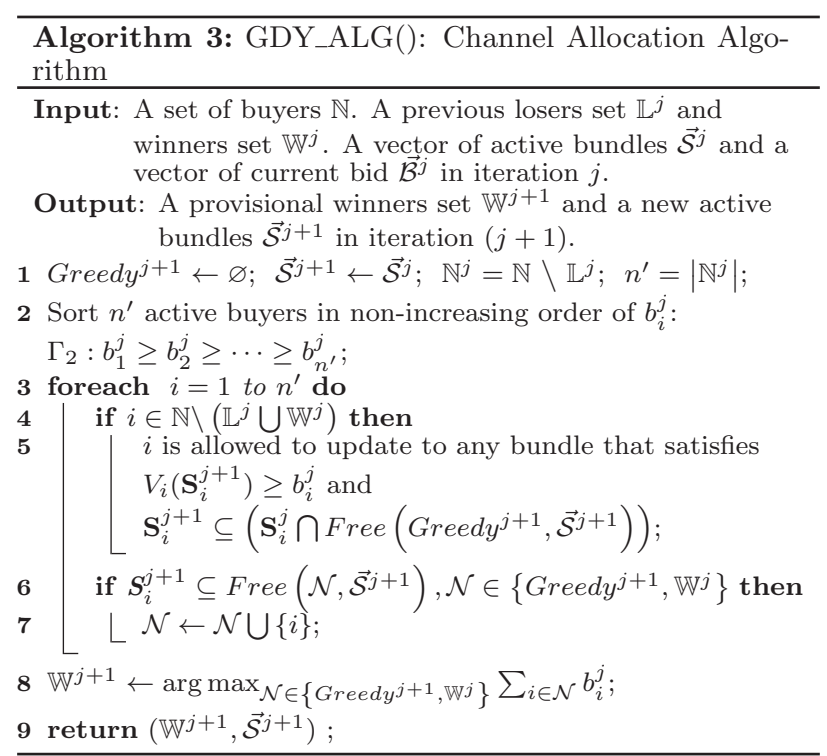

visional winners $\mathbb{W}^{j+1}$. It also outputs the updated active bundles $\overrightarrow{\mathcal{S}}^{j+1}$ (Line 8 to 9 ).

We summarize four important properties of GDY_ALG. These properties will be frequently used in next section.

- (Pareto Efficiency) For any buyer $i \notin \mathbb{W}^{j+1}, \mathbf{S}_{i}^{j+1} \nsubseteq$ $\operatorname{Free}\left(\left(\mathbb{W}^{j+1}, \overrightarrow{\mathcal{S}}^{j+1}\right)\right)$.

- (Improvement) $\sum_{i \in \mathbb{W}^{j+1}} b_{i}^{j} \geq \sum_{i \in \mathbb{W}^{j}} b_{i}^{j}$.

- (Shrinking Sets) For any buyer $i \in \mathbb{N}$ and any $0 \leq$ $j \leq J-1, \mathbf{S}_{i}^{j+1} \subseteq \mathbf{S}_{i}^{j}$.

- (First Time Shrink) Let $\mathbb{F}^{j} \triangleq\left\{i|| \mathbf{S}_{i}^{j}|=| H_{i}|,| \mathbf{S}_{i}^{j+1} \mid<\right.$ $\left.\left|H_{i}\right|\right\}$. For any $i_{1}, i_{2} \in \mathbb{F}^{j}$, it holds that $\mathbf{S}_{i_{1}}^{j+1} \cap \mathbf{S}_{i_{2}}^{j+1}=\varnothing$.

\subsection{Analysis}

In this section, we prove that AEGIS-MP is an implementation in undominated strategies by the following steps. First, we characterize the set of undominated strategies $D$. Second, we show that AEGIS-MP is individually rational for buyers taking any strategies from $D$. Third, we demonstrate that AEGIS-MP has fast undominance recognition property. Finally, we analyze the approximation ratio of AEGIS-MP.

We start with defining a type of buyers in AEGIS-MP.

Definition 8 (Drop-out if silent buyers). Active buyer $i$ is a "drop-out if silent" buyer in the jth iteration if, when she is allowed to shrink her active bundle at Line 5 in GDY_ALG, all the following hold:

- (Not a previous winner) $i \in \mathbb{N} \backslash\left(\mathbb{L}^{j} \cup \mathbb{W}^{j}\right)$.

- (Drop out if keep silent) $\mathcal{S}_{i}^{j+1} \nsubseteq$ Free $\left(\right.$ Greed $\left.{ }^{j+1}, \overrightarrow{\mathcal{S}}^{j+1}\right)$, $\overrightarrow{\mathcal{S}}_{i}^{j+1} \nsubseteq \operatorname{Free}\left(\mathbb{W}^{j}, \overrightarrow{\mathcal{S}}^{j+1}\right)$ and $e \times b_{i}^{j}>V_{i}\left(\boldsymbol{S}_{i}^{j}\right)$.

A buyer can recognize that she is a "drop-out if silent" buyer at Line 5 of GDY_ALG, and if there exists a feasible channel bundle, she will definitely shrink her active bundle. This is because she is guaranteed to drop out of the auction if she keeps silent, and if she shrinks her active bundle, she might win. In terminology of game theory, the strategy that keeping silent is dominated by the strategy of bundle shrinking.

We now characterize strategy set $D$, and claim that every strategy in $D$ is an undominated strategy.
Definition 9 (Set $D)$. Let $D$ be the set of all strategies that satisfy the following conditions, for every iteration $j$ :

- If buyer $i$ does not drop out, her bid is always less than her valuation on the active bundle, i.e., $\epsilon \leq b_{i}^{j} \leq V_{i}\left(\boldsymbol{S}_{i}^{j}\right)$. As $V_{i}\left(\boldsymbol{S}_{i}^{j}\right) \geq \epsilon$, her active bundle $\boldsymbol{S}_{i}^{j}$ must contain some interested bundles.

- If buyer $i$ drops out, then $b_{i}^{j}>V_{i}\left(\boldsymbol{S}_{i}^{j+1}\right) / e$.

- If buyer $i$ is a "drop-out if silent" buyer (Definition 8), then she will definitely declare some feasible bundle $\boldsymbol{S}_{i}^{j+1}$ that satisfies the conditions at Line 5 of GDY_ALG, if such a bundle exists.

Lemma 2. Strategy set D is a set of undominated strategies. Proof. According to the definition of $D$, all strategies outside $D$ are dominated strategies, which cannot dominate any strategy in $D$. We just need to look at any two different strategies of buyer $i$ from the set $D$ (i.e., $s t_{i}, s t_{i}^{\prime} \in D, s t_{i} \neq$ $\left.s t_{i}^{\prime}\right)$, and show that neither of them dominates the other. We consider the first point that they differ (i.e., the buyer $i$ has different active bundles). At this point, we can construct the strategies of the other buyers that will cause one strategy to win and the other to lose. Therefore, neither $s t_{i}$ nor $s t_{i}^{\prime}$ dominates the other, and then both strategies $s t_{i}$ and $s t_{i}^{\prime}$ are undominated strategies.

Lemma 3. AEGIS-MP is individually rational for buyers taking undominated strategies from $D$.

Proof. According to the fact that winners pay their lastly reported bids and the first condition of Definition 9, a winner cannot obtain a negative utility when she plays any undominated strategy in $D$. Obviously, losers' utilities are zeros. Therefore, our claim holds.

Lemma 4. AEGIS-MP has fast undominance recognition property, i.e., buyers can efficiently determine if a strategy belongs to $D$, and if not, compute an undominated strategy in $D$ to dominate it in polynomial time.

Proof. Clearly, any buyer $i$ can check if her strategy satisfies the conditions of undominated strategies in Definition 9 in polynomial time, and if not, modify her strategy to an undominated strategy that dominates the original one. $\square$

We now analyze the approximation ratio of AEGIS-MP. We first present some notations. Let $\operatorname{OPT}(\mathcal{N}, \overrightarrow{\mathcal{S}})$ denote the value of the optimal outcome (in terms of valuation function $\overrightarrow{\mathcal{V}}$ ) for a set of buyers $\mathcal{N} \subseteq \mathbb{N}$ when their channel bundles are $\overrightarrow{\mathcal{S}}$. We call $(\mathcal{N}, \overrightarrow{\mathcal{S}})$ a valid allocation, if $\mathbf{S}_{i_{1}} \bigcap \mathbf{S}_{i_{2}}=\varnothing$ for any $i_{1}, i_{2} \in \mathcal{N}$ and $\mathbf{S}_{i_{i}}, \mathbf{S}_{i_{2}} \in \overrightarrow{\mathcal{S}}$. Besides the four important properties in the previous section, we present another property, which can be derived from Definition 9 .

- (Value Bound) For any $i \in \mathbb{N}$, and any $0 \leq j \leq J$, $b_{i}^{j} \leq V_{i}\left(\mathbf{S}_{i}^{j}\right)$. For buyer $i$ who drops out in the $j$ th iteration, $b_{i}^{j}>V_{i}\left(\mathbf{S}_{i}^{j+1}\right) / e$.

Before presenting the main theorem, we give some important lemmas. We first show that the number of iterations in AEGIS-MP is limited.

Lemma 5. AEGIS-MP stops in at most $J=2 \ln \left(\bar{v}_{\max } / \epsilon\right)+$ 1 steps.

Proof. We look at a loser $i_{1}$ who drops out in the last iteration (the $(J-1)$ th iteration). According to the Pareto Efficiency property, loser $i_{1}$ 's active bundle must intersect with that of a winner $i_{2} \in \mathbb{W}^{J}$, which implies that $\mathbf{S}_{i_{1}}^{J} \cap \mathbf{S}_{i_{2}}^{J} \neq \varnothing$. Additionally, by the Shrinking Sets property, it holds that $\mathbf{S}_{i_{1}}^{j} \cap \mathbf{S}_{i_{2}}^{j} \neq \varnothing$, for any $0 \leq j \leq J-1$. Therefore, we can claim that buyer $i_{1}$ and $i_{2}$ never win together. Each of them can be a loser, and multiply her bid at most $\ln \left(\bar{v}_{\max } / \epsilon\right)$ consecutive times. We can get that $J-1 \leq 2 \ln \left(\bar{v}_{\max } / \epsilon\right)$, and thus the lemma holds. 
We have the following lemma for buyers in AEGIS-MP.

Lemma 6. For $A E G I S-M P$, it holds that $\mathrm{OPT}\left(\mathbb{L}^{J}, \overrightarrow{\mathcal{S}}^{J}\right)<$ et $J \times \mathrm{OPT}\left(\mathbb{W}^{J}, \overrightarrow{\mathcal{S}}^{J}\right)$, where $t=(1+\kappa)$.

Due to the limitation of space, we leave the detailed proof to our technical report [30].

We now present the approximation ratio of AEGIS-MP.

Theorem 3. AEGIS-MP achieves $\left(\delta_{\max }+e \delta_{\max } t J^{2}+e t J\right)$ approximation.

Proof. Let $\mathbb{O}$ be the set of winners in the optimal allocation, and winners are allocated bundles from $\overrightarrow{\mathcal{S}}^{O}=\left(\mathbf{S}_{1}^{O}, \mathbf{S}_{2}^{O}, \cdots\right.$, $\mathbf{S}_{n}^{O}$ ). We partition the winners into three categories, and bound the value of them separately.

$\triangleright$ We denote the winners that also stay in $\mathbb{W}^{J}$ by $\mathcal{N}_{1}$. In this case, winners might win other interested channel bundles in the optimal allocation, so we get

$$
\operatorname{OPT}\left(\mathcal{N}_{1}, \overrightarrow{\mathcal{S}}^{O}\right) \leq \delta_{\text {max }} \times \operatorname{OPT}\left(\mathbb{W}^{J}, \overrightarrow{\mathcal{S}}^{J}\right) .
$$

$\triangleright$ We turn to another set of winners $\mathcal{N}_{2}$, which is the subset of losers in AEGIS-MP, i.e., $\mathcal{N}_{2} \subseteq \mathbb{L}^{J}$. Buyer $i$ belongs to $\mathcal{N}_{2}$ if and only if she is a winner in the optimal allocation $\mathbb{O}$, and her allocated bundle $\mathbf{S}_{i}^{O}$ is not included in $\mathbf{S}_{i}^{J}$, which is the bundle that the buyer $i$ declares in AEGIS-MP when she drops out. We have the following claim for winners $\mathcal{N}_{2}$.

Claim 1: $\operatorname{OPT}\left(\mathcal{N}_{2}, \overrightarrow{\mathcal{S}}^{O}\right)<e \delta_{\text {max }} t J^{2} \times \mathrm{OPT}\left(\mathbb{W}^{J}, \overrightarrow{\mathcal{S}}^{J}\right)$. Proof. Let $\mathbb{F}^{j}=\left\{i \in \mathcal{N}_{2}|| \mathbf{S}_{i}^{j}|=| H_{i}|,| \mathbf{S}_{i}^{j+1}|<| H_{i} \mid\right\}$ be the set of buyers from $\mathcal{N}_{2}$ that first shrink their bundles in the $j$ th iteration, and we have $\mathcal{N}_{2}=\bigcup_{j=0}^{J-1} \mathbb{F}^{j}$. According to the first and third properties of undominated strategy in Definition 9 , we can conclude that $\mathbf{S}_{i}^{j}$ contains some interested channel bundles for all $i \in \mathbb{N}$ and $0 \leq j \leq J-1$. Therefore, for any $i \in \mathbb{F}^{j}$, we have

$$
V_{i}\left(\mathbf{S}_{i}^{O}\right) \leq \delta_{\max } \times V_{i}\left(\mathbf{S}_{i}^{J}\right), \quad \forall i \in \mathbb{F}^{j} .
$$

According to the First Time Shrink property, all bundles $\overrightarrow{\mathcal{S}}^{j+1}$ of buyers in $\mathbb{F}^{j}$ are disjoint. Additionally, by the Shrinking Sets property, we have $\mathcal{S}_{i}^{J} \subseteq \mathcal{S}_{i}^{j+1}$, implying bundles $\overrightarrow{\mathcal{S}}^{J}$ of buyers in $\mathbb{F}^{j}$ are also disjoint. Therefore, $\left(\mathbb{F}^{j}, \overrightarrow{\mathcal{S}}^{J}\right)$ is a valid allocation. Since $\mathbb{F}^{j} \subseteq \mathcal{N}_{2} \subseteq \mathbb{L}^{J}$, we get

$$
\operatorname{OPT}\left(\mathbb{F}^{j}, \overrightarrow{\mathcal{S}}^{J}\right) \leq \operatorname{OPT}\left(\mathbb{L}^{J}, \overrightarrow{\mathcal{S}}^{J}\right) \text {. }
$$

Combining with Inequalities (7)(8), we conclude that

$$
\begin{aligned}
& \operatorname{OPT}\left(\mathbb{F}^{j}, \overrightarrow{\mathcal{S}}^{O}\right)=\sum_{i \in \mathbb{F}^{j}} V_{i}\left(\mathbf{S}_{i}^{O}\right) \leq \delta_{\text {max }} \sum_{i \in \mathbb{F}^{j}} V_{i}\left(\mathbf{S}_{i}^{J}\right) \\
= & \delta_{\max } \operatorname{OPT}\left(\mathbb{F}^{j}, \overrightarrow{\mathcal{S}}^{J}\right) \leq \delta_{\max } \operatorname{OPT}\left(\mathbb{L}^{J}, \overrightarrow{\mathcal{S}}^{J}\right) .
\end{aligned}
$$

Using Inequality (9) and Lemma 6, we get

$$
\operatorname{OPT}\left(\mathbb{F}^{j}, \overrightarrow{\mathcal{S}}^{O}\right)<e \delta_{\text {max }} t J \times \mathrm{OPT}\left(\mathbb{W}^{J}, \overrightarrow{\mathcal{S}}^{J}\right) .
$$

Finally, we conclude that

$$
\operatorname{OPT}\left(\mathcal{N}_{2}, \overrightarrow{\mathcal{S}}^{O}\right) \leq \sum_{j=0}^{J-1} \operatorname{OPT}\left(\mathbb{F}^{j}, \overrightarrow{\mathcal{S}}^{O}\right)<e \delta_{\max } t J^{2} \mathrm{OPT}\left(\mathbb{W}^{J}, \overrightarrow{\mathcal{S}}^{J}\right)
$$

$\triangleright$ We denote the winners in $\mathbb{L}^{J} \backslash \mathcal{N}_{2}$ by $\mathcal{N}_{3}$. According to the definition of $\mathcal{N}_{2}$, the allocated bundles of winners in $\mathcal{N}_{3}$ are contained in bundles $\overrightarrow{\mathcal{S}}^{J}$, together with Lemma 6 , we get

$$
\begin{aligned}
& \operatorname{OPT}\left(\mathcal{N}_{3}, \overrightarrow{\mathcal{S}}^{O}\right) \leq \operatorname{OPT}\left(\mathbb{L}^{J} \backslash \mathcal{N}_{2}, \overrightarrow{\mathcal{S}}^{J}\right) \\
\leq \quad & \operatorname{OPT}\left(\mathbb{L}^{J}, \overrightarrow{\mathcal{S}}^{J}\right)<(\text { et } J) \mathrm{OPT}\left(\mathbb{W}^{J}, \overrightarrow{\mathcal{S}}^{J}\right) .
\end{aligned}
$$

We now combine these three types of winners together (Inequalities $(6)(10)(11))$, and conclude that $\mathrm{OPT}\left(\mathbb{O}, \overrightarrow{\mathcal{S}}^{O}\right) \leq$
$\operatorname{OPT}\left(\mathcal{N}_{1}, \overrightarrow{\mathcal{S}}^{O}\right)+\operatorname{OPT}\left(\mathcal{N}_{2}, \overrightarrow{\mathcal{S}}^{O}\right)+\operatorname{OPT}\left(\mathcal{N}_{3}, \overrightarrow{\mathcal{S}}^{O}\right)<\left(\delta_{\text {max }}+\right.$ $e \delta_{\text {max }} t J^{2}+$ et $\left.J\right) \mathrm{OPT}\left(\mathbb{W}^{J}, \overrightarrow{\mathcal{S}}^{J}\right)$.

From the above analysis, we now can get our main result for AEGIS-MP according to Definition 4.

Theorem 4. AEGIS-MP is an implementation of an $\mathcal{O}\left(\delta_{\text {max }} t J^{2}\right)$-approximation in undominated strategies.

\section{EVALUATION RESULTS}

In this section, we show our evaluation results. We implement AEGIS using network simulation, and compare its performance with CRWDP [5] and NSR-MP. CRWDP is an unknown single-minded combinatorial spectrum auction, and NSR-MP is a variant of AEGIS-MP. Neither CRWDP nor NSR-MP considers channel spatial reusability.

\subsection{Methodology}

We use two complementary datasets, namely Google Spectrum Database [9] and GoogleWiFi [32], to evaluate the performance of our mechanisms. We take Google Spectrum Database as our first dataset. We first extract WiFi nodes in an area (Latitude range: [40 $\left.25^{\prime} 18^{\prime \prime}, 39^{\circ} 38^{\prime} 29^{\prime \prime}\right]$, Longitude range: $\left[-76^{\circ} 34^{\prime} 40^{\prime \prime},-74^{\circ} 52^{\prime} 20^{\prime \prime}\right]$ ) from WiGLE.net [27], and we then query Google Spectrum Database the available TV white spaces and corresponding maximum permissible power for each WiFi node, which is considered as a portable device in the database. Portable devices can work on unused TV channel 21 through 51, except channel 37, 38, 39. To generate conflict graphs, we apply a simple Free Space propagation model [26] to predict the interference range between nodes, and consequently create the conflict graphs. ${ }^{3}$ We also evaluate our mechanisms in a practical conflict graph, built from exhaustive signal measurements, in the second data set. The second dataset, GoogleWiFi, records 78 APs in a $7 \mathrm{~km}^{2}$ residential area of the Google WiFi network in Mountain View, California. It was collected by a research group from UC Santa Barbara in April 2010 [32].

We build a set of auction configurations by sampling WiFi nodes in the first data set, and the number of $\mathrm{WiFi}$ nodes varies from 200 to 2000 with increment of 200 . For the second data set, we assume the number of leasing channels can be one of three values: 6,12 and 24 . We consider the case of single-minded buyers and the case of multi-minded buyers, who can have up to 10 interested bundles (i.e., $l_{i} \leq$ 10). For each buyer $i$, her $l_{i}$ interested channel bundles are randomly generated from her available channel set, and the valuations on bundles are uniformly distributed over $(0,1]$. The maximum closeness parameter of valuation is set as 5 , i.e., $\delta_{\max }=5$. The minimum monetary unit in the auction systems is set as $\epsilon=10^{-5}$. In AEGIS-MP, since buyers may have multiple undominated strategies at their decision points, we assume that buyers randomly select one of them. All the results of performance are averaged over 200 runs.

Metrics: We evaluate the following five metrics:

- Social Welfare: The sum of winning buyers'valuations on their allocated bundles of channels.

- Revenue: The sum of payments received from buyers.

- Satisfaction Ratio: The fraction of winners over buyers.

- Channel Utilization: The number of radios worked on each channel.

- Channel Eccentricity: The ratio of allocated channels over actually used channels for one buyer. In AEGIS-MP, for each winner, the final allocated bundle may contain multiple interested bundles and uninterested channels, but the winner only use one interested bundle. Therefore, we use channel eccentricity to measure this channel over-allocation.

${ }^{3}$ Other propagation models, e.g., Egli and Longley-Rice [26], could be used to generate more accurate conflict graphs. 


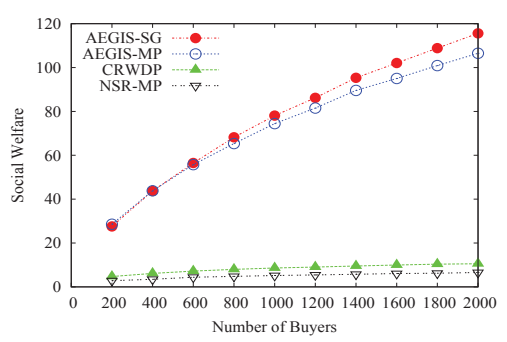

(a) Social Welfare

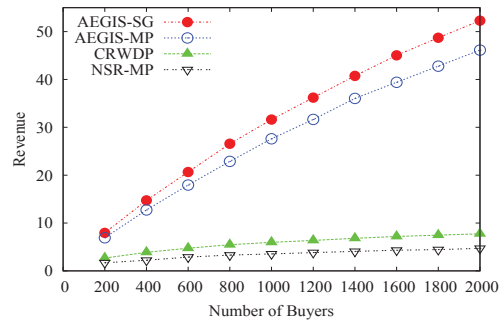

(b) Revenue

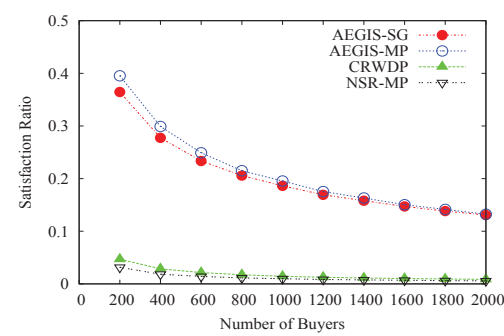

(c) Satisfaction Ratio

Figure 3: Performance of AEGIS, CRWDP and NSR-MP on Google Spectrum Dataset.

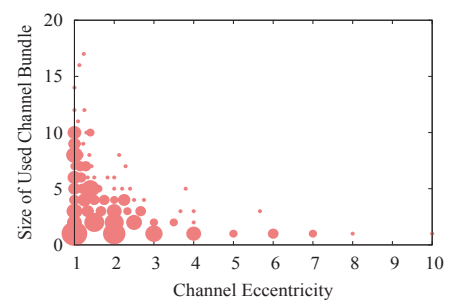

(a) Channel Eccentricity

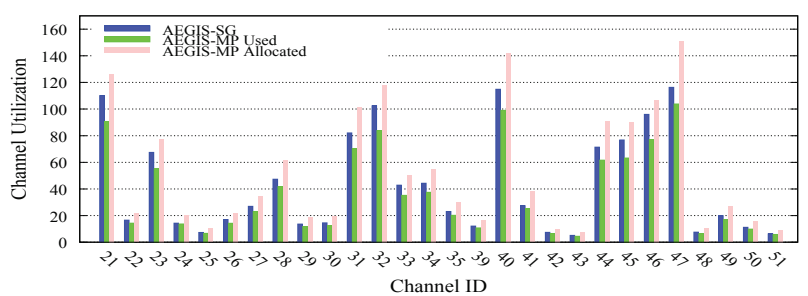

(b) Channel Utilization

Figure 4: Channel eccentricity and channel utilization of AEGIS.

\subsection{Performance on Google Spectrum Dataset}

By varying the number of buyers, we collect a set of performance data, as illustrated in Figure 3. We can see that AEGIS always outperforms the other two mechanisms, CRWDP and NSR-MP. This result demonstrates that exploiting channel spatial reusability can significantly improve the performance of spectrum auction systems. Figure 3 also shows that when the number of buyers increases, the social welfare and revenue increase, while the satisfaction ratio decreases. On one hand, AEGIS allocates channels more efficiently among more buyers, hence the social welfare and revenue increase. On the other hand, larger number of buyers leads to more intense competition on limited channels, thus decreases the satisfaction ratio. We also observe from Figure 3 that revenue is much lower than social welfare. Similar to previous work [31], we can institute reserve prices for channels to increase revenue, and make a trade-off between revenue and social welfare. How to determine an optimal reserve price is out of the scope of this paper. Intuitively, multi-minded auction mechanisms should perform better than single-minded ones because of the more feasible bundle choices for buyers. However, as shown in Figure 3, AEGIS-MP is slightly worse than AEGIS-SG in terms of social welfare and revenue. As we will discuss later, the channel eccentricity of winners in AEGIS-MP is the main reason for this degradation of system performance.

We now present the evaluation results of channel eccentricity and channel utilization. The channel eccentricity for AEGIS-SG is always equal to 1 , because the allocated bundle is exactly buyer's interested bundle. Figure 4(a) shows the channel eccentricity of AEGIS-MP. We randomly select one instance from the 200 simulation instances when the number of buyers is fixed at 2000, and calculate the channel eccentricity for each winner. The placement of a circle in Figure 4(a) indicates one set of winners with the same channel eccentricity and the same size of used channel bundle. The size of a circle is logarithmic to the number of winners. Though some of winners' channel eccentricities are equal to 1 , there exist about $67 \%$ winners, whose channel eccentricities are larger than 1. On one hand, AEGIS-MP just stimulates buyers to take undominated strategies, such that buyers can still maintain multiple bundles or uninterested channels in their active bundles during the auction. On the other hand, buyers only use the most valuable channel subset among their allocated bundles. Therefore, the size of allocated bundle can be larger than that of actually used bundle in some cases, leading to channel over-allocation.

The channel eccentricity affects the channel utilization of AEGIS-MP. By fixing the number of buyers at 2000 and running 200 simulation instances, we record the average channel utilization for each channel, and plot the results in Figure 4(b). We do not include CRWDP and NSR-MP in this analysis, because they do not consider channel spatial reusability. As shown in the figure, different TV channels have different channel utilization. The reason is that TV white spaces are spatially heterogeneous, e.g., channel 47 can be accessed to almost all buyers, while channel 33 are only available to around $36 \%$ buyers. In AEGIS-MP, we distinguish between allocated channels and used channels. We can observe from Figure 4(b) that the allocated number is always larger than the used number for each channel. This is because some winners have channel eccentricity higher than 1. We can also see from Figure 4(b) that the channel utilization of AEGIS-MP is always lower than that of AEGIS-SG. The reason is that the winners with high channel eccentricity in AEGIS-MP disables some possible allocations of their interfering neighbors. From the above analysis, we can get that buyers' manipulated strategies on channel demands indeed impact the performance of spectrum auction systems.

\subsection{Performance on GoogleWiFi Dataset}

Figure 5 shows the system performance of AEGIS, CRWDP, and NSR-MP on GoogleWiFi dataset when there are $6,12,24$ channels. Since channels are accessible to all buyers in this setting, we average the channel utilization on all channels on this dataset. Generally, the evaluation re- 


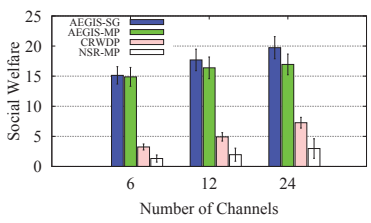

(a) Social Welfare

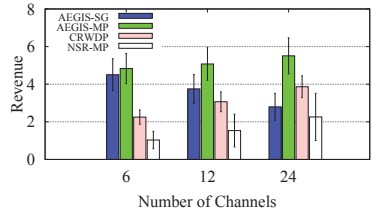

(b) Revenue

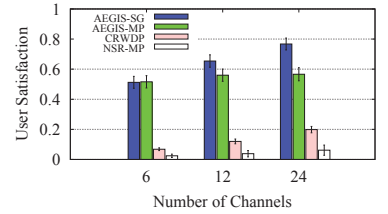

(c) Satisfaction Ratio

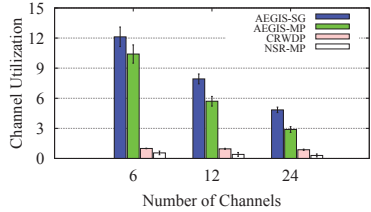

(d) Channel Utilization

Figure 5: Performance of AEGIS, CRWDP and NSR-MP on GoogleWiFi Dataset.

sults are similar with those on Google Spectrum Dataset. Again, AEGIS achieves better performance than CRWDP and NSR-MP. Figure 5 also shows that when the number of channels increases, the social welfare and satisfaction ratio increase, and channel utilization decreases. The reason is that fixing the number of buyers, larger supply of channels results in more trades in the auction, and thus increases social welfare and satisfaction ratio. The channel utilization decreases because buyers can be allocated to more channels when the number of channels increases. For revenue, AEGIS-SG decreases with the number of channels, while AEGIS-MP, CRWDP and NSR-MP increase. The clearing price calculation in AEGIS-SG is based on critical bid. When larger number of channels are accessible in the auction, more buyers are allocated channels, reducing the critical bids for winners. Hence, the revenue of AEGIS-SG decreases. Though the clearing price of CRWDP is also calculated based on critical bid, there still exist considerable losers when the number of channels becomes large. Therefore, the critical bids for winners still stay high, so that the revenue continue to grow with the increase of channels. The clearing prices of AEGIS-MP and NSR-MP are the bids of winners at the end of the auctions. Larger supply of channels leads to more winners, and thus revenues in AEGIS-MP and NSR-MP become higher.

\section{CONCLUSION}

Considering the five challenges for designing a practical spectrum auction mechanism, we have proposed AEGIS, which is the first framework of unknown combinatorial auction mechanisms for heterogeneous spectrum redistribution. For the case with unknown single-minded buyers, we have designed a direct revelation combinatorial auction mechanism, call AEGIS-SG. AEGIS-SG achieves strategy-proofness and approximately efficient social welfare. We have further considered the case with unknown multi-minded buyers, and designed an iterative ascending combinatorial auction, namely AEGIS-MP. AEGIS-MP is implemented in undominated strategies, and has a good approximation ratio. We have implemented AEGIS and evaluated its performance on two practical datasets. Compared with the existing work, AEGIS achieves superior performance, in terms of social welfare, revenue, satisfaction ratio, and channel utilization.

\section{REFERENCES}

[1] A. Archer and E. Tardos. Truthful mechanisms for one-parameter agents. In FOCS, 2001

[2] M. Babaioff, R. Lavi, and E. Pavlov. Single-value combinatorial auctions and algorithmic implementation in undominated strategies. Journal of the ACM, 56(1):4:1-4:32, 2009.

[3] P. Briest, P. Krysta, and B. Vöcking. Approximation techniques for utilitarian mechanism design. In STOC, 2005

[4] S. Dobzinski. An impossibility result for truthful combinatorial auctions with submodular valuations. In STOC, 2011.

[5] M. Dong, G. Sun, X. Wang, and Q. Zhang. Combinatorial auction with time-frequency flexibility in cognitive radio networks. In INFOCOM, 2012
[6] Federal Communications Commission (FCC). http://www.fcc.gov/

[7] X. Feng, Y. Chen, J. Zhang, Q. Zhang, and B. Li. TAHES: Truthful double auction for heterogeneous spectrums. In INFOCOM, 2012

[8] L. Gao, Y. Xu, and X. Wang. MAP: Multiauctioneer progressive auction for dynamic spectrum access. IEEE Transactions on Mobile Computing, 10(8):1144-1161, 2011.

[9] Google Spectrum Database. https://www.google.com/get/spectrumdatabase/.

[10] M. Hoefer and T. Kesselheim. Secondary spectrum auctions for symmetric and submodular bidders. In EC, 2012.

[11] Radio Regulations, International Telecommunication Union, Genève, 2012.

[12] M. O. Jackson. Implementation in undominated strategies: A look at bounded mechanisms. The Review of Economic Studies, 59(4):757-775, 1992.

[13] K. Jagannathan, I. Menache, G. Zussman, and E. Modiano. Non-cooperative spectrum access: The dedicated vs. free spectrum choice. In MobiHoc, 2011.

[14] Japanese Auction. http://en.wikipedia.org/wiki/Japanese_auction.

[15] J. Jia, Q. Zhang, Q. Zhang, and M. Liu. Revenue generation for truthful spectrum auction in dynamic spectrum access. In MobiHoc, 2009.

[16] P. Klemperer. How (not) to run auctions: The european $3 g$ telecom auctions. European Economic Review, telecom auctions. Europe

[17] D. Lehmann, L. I. Oćallaghan, and Y. Shoham. Truth revelation in approximately efficient combinatorial auctions. Journal of the ACM, 49(5):577-602, 2002.

[18] LTE Spectrum and Network Strategies. http://www.adlittle.com/downloads/tx_adlreports/ADL_LTE_ Spectrum_Network_Strategies.pdf.

[19] A. Mas-Colell, M. D. Whinston, and J. R. Green. Microeconomic Theory. Oxford Press, 1995.

[20] A. Mu'alem and N. Nisan. Truthful approximation mechanisms for restricted combinatorial auctions. Games and Economic Behavior, 64(2):612 - 631, 2008.

[21] N. Nisan. Bidding and allocation in combinatorial auctions. In $E C, 2000$.

[22] M. J. Osborne and A. Rubenstein. A Course in Game Theory. MIT Press, 1994

[23] C. Papadimitriou, M. Schapira, and Y. Singer. On the hardness of being truthful. In FOCS, 2008.

[24] S.-P. Sheng and M. Liu. Profit incentive in a secondary spectrum market: A contract design approach. In INFOCOM, 2013.

[25] Spectrum Bridge. http://www.spectrumbridge.com/Home.aspx.

[26] D. Tse and P. Viswanath. Fundamentals of Wireless Communication. Cambridge University Press, May 2005.

[27] WiGLE. https://wigle.net/.

[28] P. Xu, S. Wang, and X.-Y. Li. SALSA: Strategyproof online spectrum admissions for wireless networks. IEEE Transactions on Computers, 59(12):1691 -1702, 2010.

[29] Z. Zheng, F. Wu, and G. Chen. SMASHER: A strategy-proof combinatorial auction mechanism for heterogeneous channel redistribution. In MobiHoc, 2013.

[30] Z. Zheng, F. Wu, S. Tang, and G. Chen. Unknown combinatorial auction mechanisms for heterogeneous spectrum redistribution. Technical report, 2014. available at http://www.cs.sjtu.edu.cn/ ${ }^{f}$ wu/res/Paper/ZWTC14TRAEGIS.pdf.

[31] X. Zhou, S. Gandhi, S. Suri, and H. Zheng. eBay in the sky: Strategy-proof wireless spectrum auctions. In MobiCom, 2008.

[32] X. Zhou, Z. Zhang, G. Wang, X. Yu, B. Y. Zhao, and H. Zheng. Practical conflict graphs for dynamic spectrum distribution. In SIGMETRICS, 2013.

[33] X. Zhou and H. Zheng. TRUST: A general framework for truthful double spectrum auctions. In INFOCOM, 2009. 